\title{
Cloning and expression of two new prolactin-related proteins, prolactin-related protein-VIII and -IX, in bovine placenta Koichi Ushizawa ${ }^{1}$, Toru Takahashi ${ }^{1}$, Misa Hosoe ${ }^{1}$, Kanako Kaneyama ${ }^{1,3}$ and Kazuyoshi Hashizume*2
}

\author{
Address: ${ }^{1}$ Reproductive Biology and Technology Laboratory, Developmental Biology Department, National Institute of Agrobiological Sciences, 2 \\ Ikenodai, Tsukuba, Ibaraki 305-8602, Japan, 2Department of Veterinary Medicine, Faculty of Agriculture, Iwate University, 3-18-8 Ueda, Morioka, \\ Iwate 020-8550, Japan and ${ }^{3}$ Department of Technology, National Livestock Breeding Center, 1 Odakurahara, Odakura, Nishigo, Fukushima 961- \\ 8511, Japan \\ Email: Koichi Ushizawa - ushizawa@affrc.go.jp; Toru Takahashi - tatoru@affrc.go.jp; Misa Hosoe - hosoe@affrc.go.jp; \\ Kanako Kaneyama - k0kaneym@nlbc.go.jp; Kazuyoshi Hashizume* - kazuha@iwate-u.ac.jp \\ * Corresponding author
}

Published: 07 December 2005

Reproductive Biology and Endocrinology 2005, 3:68 doi:10.1186/1477-7827-3-68

This article is available from: http://www.rbej.com/content/3/I/68

(C) 2005 Ushizawa et al; licensee BioMed Central Ltd.

This is an Open Access article distributed under the terms of the Creative Commons Attribution License (http://creativecommons.org/licenses/by/2.0), which permits unrestricted use, distribution, and reproduction in any medium, provided the original work is properly cited.
Received: 07 October 2005

Accepted: 07 December 2005

\begin{abstract}
Background: Prolactin-related proteins (PRPs) are specific proteins of the growth hormone/ prolactin (GH/PRL) family in bovine placenta. This study reports the identification and sequencing of a full-length cDNA for two new members of bovine PRPs, bPRP-VIII and -IX, and their localization and quantitative expression in bovine placenta.
\end{abstract}

Methods: New bPRP-VIII and -IX were identified from bovine placentome. Localization and quantitative gene expression in the placenta were respectively investigated by in situ hybridization and real-time RT-PCR methods. Recombinant proteins of these genes were produced by a mammalian HEK293 cell expression system.

Results: Full-length bPRP-VIII and -IX cDNA were respectively cloned with 909 and 910 nucleotide open-reading-frames corresponding to proteins of 236 and 238 amino acids. The predicted bPRP-VIII amino acid sequence shared about 40 to $70 \%$ homology with other bPRPs, and bPRP-IX had about 50 to $80 \%$ homology of others. The two new bPRPs were detected only in the placenta by RT-PCR. mRNA was primarily expressed in the cotyledon and intercotyledonary tissues throughout gestation. An in situ hybridization analysis revealed the presence of bPRP-VIII and -IX mRNA in the trophoblastic binucleate and/or trinucleate cells. bPRP-VIII mRNA was observed in the extra-embryonic membrane on Day 27 of gestation, however, no bPRP-IX mRNA was observed in the extra-embryonic membrane in the same stage of pregnancy by quantitative real-time RT-PCR analysis. Both new bPRP genes were possible to translate a mature protein in a mammalian cell expression system with approximately $28 \mathrm{kDa}$ in bPRP-VIII and $38 \mathrm{kDa}$ in bPRP-IX.

Conclusion: We identified the new members of bovine prolactin-related protein, bPRP-VIII and IX. Localization and quantitative expression were confirmed in bovine placenta by in situ hybridization or real-time PCR. Their different temporal and spatial expressions suggest a different role for these genes in bovine placenta during gestation. 


\section{Background}

Prolactin-related proteins (PRPs) are members of the growth hormone/prolactin (GH/PRL) family [1]. PRPs are highly expressed in binucleate cells of bovine trophoblasts, although their function is still obscure. No new member had been identified between early 1990 and this year [2]. We recently found a new $P R P$ member through a comprehensive analysis using a bovine utero-placental cDNA microarray [3]. Seven bovine PRP (bPRP) genes have been identified in placentomal tissues, whereas only two of those genes have been shown to be translated. The expression pattern of these genes is spatially and temporally different. $b P R P-I$ indicates the considerable importance of regulation of implantation and formation of the placentome in bovines $[4,5]$. However, bPRP-VII was expressed in intercotyledon and cotyledon with rather weak compare to those of $b P R P-I$ during the gestation [6].

Placental lactogens (PLs) are known as classical members and PRPs are categorized as the non-classical members of the GH/PRL family in bovine [7]. Various non-classical members are common in rodents, such as mice and rats, and some of them exhibit known biological functions, such as proliferin and prolactin-like protein-A (PLP-A) [712]. However, as a whole the biological function of Prolactin-related genes is still not known in various species including bovine. Two research groups identified bovine PRPs mRNA individually; $b P R P-I$ to $-I I I$ were identified by the Schuler group $[13,14]$, and $b P R P-I V$ to $-V I$ were identified by the Nakashima group [15-17]. We recently identified another one named as bPRP-VII [6]. Our previous study suggested our bovine placental cDNA library contained other $b P R P$ genes [3]. We reveal here the full-length sequences of two new members of $b P R P s, b P R P$-VIII and $I X$, and their localization and quantitative expression. We confirmed the possibility of translation of them and the accuracy of these gene sequences to produce a recombinant protein using the HEK293 cell-transfecting system.

\section{Materials and methods Animals and tissues}

Placental tissues for cDNA cloning and mRNA expression were collected from Japanese Black cows. The extraembryonic tissues, placenta, and endometrium were collected at a local slaughterhouse on days 27 to 28,56 to 64 , 144 to 149 , and 245 to 258 after artificial insemination (day 1). The tissues were separated into four portions: the cotyledon (COT); intercotyledon (the area between the cotyledonary villous (ICOT)); the caruncle area, including the maternal placentomal septa in the endometrium (CAR); and the intercaruncle area (ICAR). It was difficult to divide the COT and ICOT on days 27 to 28 , and thus the COT contained very few villi. Tissues from two different cows on day 27 and one cow on day 28 of gestation (n = 3) were used as Day 27 extra-embryonic membrane
(Day 27EEM), Day 27 caruncle (Day 27CAR), and Day 27 intercaruncular endometrium (Day 27ICAR). Placentomal tissues were collected on days 56, 58, and 64 (in total, $\mathrm{n}=3$ ) and designated as Day 60COT, CAR, ICOT, and ICAR. Sample materials from days 144, 148, and 149 $(\mathrm{n}=3)$ and days 245 (two samples) and 252 (one sample) were marked as Day 150COT, CAR, ICOT, ICAR, Day 250COT, CAR, ICOT, and ICAR. The cotyledonary and caruncular part was separated mechanically, and each part may contained a counter part of tissue. The collected samples were stored at $-80^{\circ} \mathrm{C}$ until RNA extraction. The placentomes of day 60 were fixed in $3.7 \%$ formaldehyde PBS at $\mathrm{pH} 7.4$ and then embedded in paraffin wax and stored at $4^{\circ} \mathrm{C}$ until in situ hybridization. All procedures for these animal experiments were carried out in accordance with the guidelines and ethics approved by the Animal Ethics Committee of the National Institute of Agrobiological Sciences for the use of animals.

\section{Cloning of full-length bPRP-VIII and -IX CDNA}

The new full-length $b P R P$-VIII and -IX CDNA were isolated from bovine cotyledonary tissue by the 3'-rapid amplification of cDNA ends (RACE) method. In brief, a complete RNA was isolated from a bovine placentome on day 60 of gestation using ISOGEN (Nippon Gene, Toyama, Japan). We performed 3'-RACE using a 3'-full RACE core set (Takara, Kyoto, Japan) with bPRP-VIII-specific forward primer (5'-CCACAGTCAACAGGAGTCCTCA-3') and bPRP-IX-specific forward primer (5'-CCAACAGAGAGTCCTCACCCTGCGA-3'). The bPRP-VIII and -IX primer was designed from bovine EST accession number AW464912 and BP108069, respectively. The 3'-RACE products were sequenced using an ABI Prism 370 automatic sequencer (Applied Biosystems, Foster City, CA, USA) after cloning into a pGEM-T Easy vector (Promega, Madison, WI, USA).

\section{Phylogenetic analysis}

Alignments of deduced protein sequences were performed with the multiple alignment software Clustal W 1.83 on the DDBJ web site. Clustal W was also employed to calculate trees using the Neighbor-Joining (NJ) method [18]. TreeView was used to display the phylogenetic tree [19]. The values represent bootstrap scores for 1,000 trials, indicating the credibility of each branch. Except the bPRP-VIII and -IX sequences, all the bPRPs and bPLs protein sequences were obtained from GenBank. Their GenBank accession numbers are: bPRP-I ([02944), bPRP-II

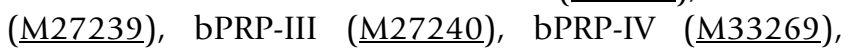
bPRP-V (AB239755), bPRP-VI (X59504), bPRP-VII (AB187564), bPL-Ala (J02840), and bPL-Val (M33268).

\section{Three-dimensional structure prediction by FAMS}

We predicted the three-dimensional (3D) structure of bPRP-VIII and -IX by using the FAMS (Fully automated homology modeling system; http://www.pharm.kitasato- 

47
ATG GCT CCA GCT CCC AGC TTC TGT GGA CAC CAG TGG ACT TAC AAC CCT GTC CAA GGG TCC Met Ala Pro Ala Pro ser phe Cys Gly His Gln Trp Thr Tyr Asn Pro Val Gln Gly Ser 1

107

CTG CTG CTG CTG CTG GTC ATG TCA AAT CTG CTC CTG TGC CAA GGC AGA ACA TGC CCG Cys Leu Leu Leu Leu Leu Val Met Ser Asn Leu Leu Leu Cys Gln Gly Arg Thr Cys Pro 21

167

TTC TGC TGT TCT GAC GTG TTT GAC ATC CCC TTG GAA TCC CTT AGA AAC CTG TTT CTC AAT Phe Cys Cys Ser Asp Val Phe Asp Ile Pro Leu Glu Ser Leu Arg Asn Leu Phe Leu Asn 41

GCT ACC ATG TTG TCC CGC GAC ATT GCC ATC CAT TCC AGC ATA ATG TTC ACT GAT TTT GAT

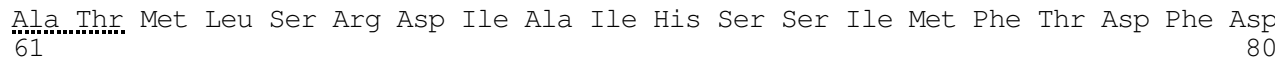

GAA AAA TAT GCC CAG GGC AAA CAG TAC GAT ATC AAT GAC AAG AGC TGC CAT ACC GAT CCC Glu Lys Tyr Ala Gln Gly Lys Gln Tyr Asp Ile Asn Asp Lys Ser Cys His Thr Asp Pro 81

100

347 406

CTC CCT ACT CCC GAA GAA AGA GAA CAA GCC CTA AAG ATA AAC AAT GAA GAC CTG AGT AAG Leu Pro Thr Pro Glu Glu Arg Glu Gln Ala Leu Lys Ile Asn Asn Glu Asp Leu Ser Lys 101

120

407

466

TGG ATA ATC ATG TTA CTG TAC TCC TGG AAA AGA CCT CTA AAT CAT CTA GTC ACA GAG CTG Trp Ile Ile Met Leu Leu Tyr Ser Trp Lys Arg Pro Leu Asn His Leu Val Thr Glu Leu 121

140

467

526

CAG AAT ATG AAA GAA GTC TCA GAT ACT ATC CTA TCA AGT GCC AGA CAA AAT TTG AAA AAA

Gln Asn Met Lys Glu Val Ser Asp Thr Ile Leu Ser Ser Ala Arg Gln Asn Leu Lys Lys 141

160

527586

GTA CAA GAA CTT CAA GAA TTC ATA GAG AGG CAA TTC AGC CAG ATT ATT TTT CCA GTA AGG Val Gln Glu Leu Gln Glu Phe Ile Glu Arg Gln Phe Ser Gln Ile Ile Phe Pro Val Arg 161

587

180

CGG CTG ACC GAG GGT GAA ATA GTC TGG TCA GAA CTT CCA TCC CTG GTG TCC AGC GA Arg Leu Thr Glu Gly Glu Ile Val Trp Ser Glu Leu Pro ser Leu Val Ser Ser Asp Glu 181

647 GAT ATT CGT CAT TCT GCA TTT TAT AAC CTG TTC AAG TGC CTG CAG AGG GAT TCA CAT AAA Asp Ile Arg His Ser Ala Phe Tyr Asn Leu Phe Lys Cys Leu Gln Arg Asp Ser His Lys 201

707

GTT GAC ATG TAC ACC AAG ATC ATG TTG TGC CGA ATC AAC AAC ACG TGC TAA

Val Asp Met Tyr Thr Lys Ile Met Leu Cys Arg Ile Asn Asn Thr Cys *** 221

758 236

ATCCAAAGCATCCCATCCAGCTCTGAgGTGGTGGTAATGTTCTATCCCATAGCAAGCTTCTTAGAAATTTATAGTTTTI

$837 \quad 906$

TAATGCATGCTCTGTGAAATGGGTCTCCTCTAAAAAAATAAACACAGACTCTGTAGAAAAAAAAAAAAAA

\section{Figure I}

Nucleotide and deduced amino acid sequences of bPRP-VIII. The arrow indicates the putative primary cleavage site of the signal peptide. The potential $\mathrm{N}$-glycosylation site is underlined with a dotted line. The asterisks indicate the termination codon. The polyadenylation signal is underlined with a solid line. 
Table I: Oligonucleotide primers used for RT-PCR

\begin{tabular}{|c|c|c|c|}
\hline Gene & Primer & Sequence & Position \\
\hline bPRP-VIII & Forward & 5'-AGAACATGCCCGTTCTGCTGT-3' & $155-175$ \\
\hline$(A B \mid 96438)$ & Reverse & 5'-TTAGCACGTGTTGTTGATTCG-3' & $757-737$ \\
\hline$b P R P-I X$ & Forward & 5'-AACTCATGCCCATCCTGTGGT-3' & $150-170$ \\
\hline$(\mathrm{AB} 20488 \mathrm{I})$ & Reverse & 5'-TTAGCACTTTTTGCGGATTCG-3' & $758-738$ \\
\hline GAPDH & Forward & 5'-CСTTCATTGACCTTCACTACATGGTCTA-3' & $71-98$ \\
\hline (U85042) & Reverse & 5'-GCTGTAGCCAAATTCATTGTCGTACCA-3' & $927-901$ \\
\hline
\end{tabular}

u.ac.jp/fams/index.html) [20]. FAMS is the software which predicts 3D model of the target protein from the structural known protein of high homology. In case of bPRP-VIII and -IX, the 3D structure was constructed based on the human prolactin (hPRL) 3D structure (Protein Data Bank ID: 1N9D) in the element. FAMS program only requires an amino acids sequence as input, and constructs 3D model structures automatically. Visualization of the 3D structure was performed using the RasMol 2.7.3 software http://www.bernstein-plus-sons.com/software/ras mol/[21].

\section{RT-PCR}

Tissue distribution of $b P R P$-VIII and -IX expression was studied by RT-PCR. Bovine GAPDH was used as a positive control for the PCR. Details of the RT-PCR method were described in previous reports $[6,22]$. The total RNA in a total reaction mixture was used for reverse transcription and template cDNA synthesis using oligo(dT) primer and Superscript II reverse transcriptase (Invitrogen, Carlsbad, CA, USA) at $42^{\circ} \mathrm{C}$ for $50 \mathrm{~min}$. Each PCR contained the cDNA template, primers, deoxynucleotide triphosphate mixture (dNTP), $\mathrm{MgCl}_{2}, 10 \times$ PCR buffer II, autoclaved milliQ water, and AmpliTaq gold DNA polymerase (Applied Biosystems). Amplification conditions included denaturation at $95^{\circ} \mathrm{C}$ for $30 \mathrm{sec}$ and extension at $72^{\circ} \mathrm{C}$ for $1 \mathrm{~min}$. Twenty-seven cycles were performed for all samples. The annealing temperature was set at $60^{\circ} \mathrm{C}$ for 30 sec. A single denaturation step at $95^{\circ} \mathrm{C}$ for $10 \mathrm{~min}$ before the first PCR cycle and a final extension step at $72^{\circ} \mathrm{C}$ for $10 \mathrm{~min}$ after the last PCR cycle were also performed. The PCR products were analyzed by agarose gel electrophoresis and visualized by ethidium bromide staining. The primers encoding for the $b P R P$-VIII and -IX sequences were designed using the sequence illustrated in Fig. 1. The primer sequence was selected to include a mismatch for other bPRPs. The designated primers are listed in Table 1. All the primers were commercially synthesized (Espec Oligo Service, Tsukuba, Japan).

\section{In situ hybridization}

Full-length cDNA of bPRP-VIII and -IX was used as a template for hybridization probe synthesis. Digoxigenin (DIG)-labeled antisense and sense-complementary RNA probes were prepared as described in previous studies $[6,23]$. The placentomes were sectioned into $7 \mu \mathrm{m}$-thick sections for hybridization. In situ hybridization was performed using automated Ventana HX System Discovery with a RiboMapKit and BlueMapKit (Ventana, Tucson, AZ, USA) [6]. Briefly, sections were hybridized with DIGlabeled probes in RiboHybe (Ventana) hybridization solution at $65^{\circ} \mathrm{C}$ for 6 hours. The sections were washed three times in RiboWash (Ventana) $\left(65^{\circ} \mathrm{C}, 6 \mathrm{~min}\right)$ after hybridization and were fixed in RiboFix (Ventana) $\left(37^{\circ} \mathrm{C}\right.$, $10 \mathrm{~min})$. The hybridization signals were then detected with monoclonal-anti-digoxin biotin conjugate (Sigma, Saint Louis, MI, USA). The hybridized glasses were observed after preparation with a Nikon ECLIPSE E800 photomicroscope (Nikon, Tokyo, Japan).

\section{Real-time RT-PCR}

Gene expression of $b P R P$-VIII and -IX was confirmed quantitatively at each stage of gestation (Days 27FM, 27CAR, 27ICAR, 60COT, 60CAR, 60ICOT, 60ICAR, 150COT, 150CAR, 150ICOT, 150ICAR, 250COT, 250CAR, 250ICOT, and 250ICAR) by real-time RT-PCR analysis. Details of the real-time RT-PCR procedures have been described in previous reports $[6,24]$. Briefly, fifty ng of the total RNA were reverse transcribed into cDNA for 30 min at $48^{\circ} \mathrm{C}$ by MultiScribe ${ }^{\mathrm{TM}}$ reverse transcriptase with an Oligo dT primer, dNTP mixture, $\mathrm{MgCl}_{2}$ and RNase inhibitor. Primer pairs and oligonucleotide probes labeled with a reporter fluorescent dye at the 5 '-end and a quencher fluorescent dye at the 3 '-end were designed using the Primer Express computer software program (Applied Biosystems). The primers and probes for each gene are listed in Table 2. The thermal-cycling conditions included initial sample incubation at $50^{\circ} \mathrm{C}$ for $2 \mathrm{~min}$ and at $95^{\circ} \mathrm{C}$ for 10 min, followed by 40 cycles at $95^{\circ} \mathrm{C}$ for $15 \mathrm{sec}$ and at $60^{\circ} \mathrm{C}$ for $1 \mathrm{~min}$. The cycle threshold values $\left(\mathrm{C}_{\mathrm{T}}\right)$ indicate the quantity of the target gene in each sample and were determined in real time using an ABI Prism 7700 sequence detector (Applied Biosystems). The relative difference in the initial amount of each mRNA species (or CDNA) was determined by comparing the $\mathrm{C}_{\mathrm{T}}$ values. The standard curves for each gene were generated by serial dilution of plasmid containing bPRP-VIII, -IX, or GAPDH cDNA to quantify the mRNA concentrations. The ratio of $b P R P-V I I I$ 
Table 2: Oligonucleotide primers and TaqMan probes used for real-time RT-PCR analysis

\begin{tabular}{|c|c|c|c|}
\hline Gene & Primer or TaqMan probe & Sequence & Position \\
\hline bPRP-VIII & Forward & 5'-CAAGGGTCCTGCCTGCTG-3' & $98-115$ \\
\hline \multirow[t]{2}{*}{$(A B \mid 96438)$} & Reverse & 5'-GGCATGTTCTGCCTTGGC-3' & $164-344$ \\
\hline & Probe & 5'-TGCTGCTGGTCATGTCAAATCTGCTCC-3' & $117-143$ \\
\hline bPRP-IX & Forward & 5'-ATATGCCCAGGGCAAACTGT-3' & $287-306$ \\
\hline \multirow[t]{2}{*}{ (AB20488I) } & Reverse & 5'-TCGGGAGCATGGAAGGAAT-3' & $358-340$ \\
\hline & Probe & 5'-TATCAATGCCACCAACAGCTGCCACA-3' & $311-336$ \\
\hline GAPDH & Forward & 5'-AAGGCCATCACCATCTTCCA-3' & $178-197$ \\
\hline \multirow[t]{2}{*}{ (U85042) } & Reverse & 5'-CCACTACATACTCAGCACCAGCAT-3' & $253-230$ \\
\hline & Probe & 5'-AGCGAGATCCTGCCAACATCAAGTGG-3' & $200-225$ \\
\hline
\end{tabular}

TaqMan probes of bPRP-VIII and -IX have 5'-FAM dye and 3'-TAMRA dye

TaqMan probe of GAPDH has 5'-VIC dye and 3'-TAMRA dye

and -IX mRNA to GAPDH mRNA was calculated to adjust for any variations in the RT-PCR reaction. All values are presented as mean \pm SD. Statistical analysis was performed using one-way ANOVA followed by the TukeyKramer multiple comparison test. Differences were considered significant at $P<0.05$.

\section{Production of recombinant proteins}

The bPRP-I, -VIII, -IX, and bPL sequences encoding the mature protein region, which combined the FLAG and $6 \times$ His epitope tag sequences, were inserted into the pFLAGCMV-3 vector (Sigma). The constructed plasmid was transfected into HEK 293 cells using FuGENE 6 (Roche Diagnostics, Basel, Switzerland) for transient transfection. Stably transfected HEK 293 cells were adapted to the suspension culture in a spinner flask using 293 SFM II medium (Invitrogen, Gibco) and cultured in an atmosphere of $5 \% \mathrm{CO}_{2}$ in air at $37^{\circ} \mathrm{C}$ for 3 days. The medium was separated by centrifugation and stored at $-30^{\circ} \mathrm{C}$.

\section{Western blot analysis}

The $10 \mu \mathrm{g}$ of proteins from the HEK293 cell conditioned media were loaded on each lane, separated by SDS-PAGE, and electrophoretically transferred onto a polyvinylidene difluoride membrane [25]. Western blotting was performed by the method of Towbin et al. [26]. Briefly, the membrane was blocked in $10 \%$ skim milk overnight, incubated with anti-FLAG M2 (Sigma) for $1 \mathrm{~h}$ at room temperature, followed by incubation with anti-mouse IgG conjugated with alkaline phosphatase (Sigma) (diluted 1:3000) for $1 \mathrm{~h}$ at room temperature. Immunopositive bands were stained using NBT (Bio-Rad, Hercules, CA, USA) and BCIP (Bio-Rad).

\section{Results}

Sequences of bPRP-VIII and -IX cDNA

Full-length $b P R P$-VIII and -IX were cloned from bovine placentome. The 906- and 910-nucleotide sequences were isolated in bPRP-VIII and -IX, respectively (Fig. 1 and 2). The protein sequence regions (CDSs) were composed of
711 nucleotides in $b P R P$-VIII and 717 nucleotides in bPRP-IX. The 3'-untranslated region contains one AATAAA polyadenylation signal start 20 and 26 bases upstream from the poly (A) addition site in bPRP-VIII and -IX, respectively. The amino acid sequences deduced from fulllength $b P R P$-VIII and $b P R P-I X$ CDNA are amino acids 236 and 238. The homology of predicted amino acid sequences of bPRP-VIII and -IX protein were shown in Fig. 3. The predicted sequence of bPRP-VIII protein was $69 \%$ homologous to that of bPRP-VI, 66\% homologous to that of bPRP-VII, 61\% homologous to that of bPRP-I and -III, $58 \%$ homologous to that of bPRP-IV and -V, 57\% homologous to that of bPRP-IX, 42\% homologous to that of bPRP-II, and 39\% homologous to that of bPL-Ala (Fig. 3). The predicted sequence of bPRP-IX protein was $81 \%$ homologous to that of bPRP-IV, 76\% homologous to that of bPRP-I, $70 \%$ homologous to that of bPRP-II, $60 \%$ homologous to that of bPRP-VII, 57\% homologous to that of bPRP-VI and -VIII, 53\% homologous to that of bPRP-III and -V, and 40\% homologous to that of bPL-Ala (Fig. 3). In the phylogenetic analysis, it was shown that bPRP-VIII was close to bPRP-III, bPRP-VI, and bPRP-VII sides in the phylogenetic tree and bPRP-IX was close to bPRP-II and bPRP-IV sides in the phylogenetic tree (Fig. 4 ). The $N$-terminal regions of the bPRP-VIII and -IX proteins were rich in hydrophobic amino acid residue, which is characteristic of the signal peptide. bPRP-VIII had two consensus sequences for N-glycosylation and Asn-X-Ser/ Thr at the positions of 60 to 62 and 233 to 235 (Fig. 1). bPRP-IX also had four consensus sequences for N-glycosylation at the positions of 70 to 72,92 to 94,146 to 148 , and 160 to 162 (Fig. 2). Another atypical $N$-glycosylation site, Asn-X-Cys was found in only bPRP-IX at the position of 95 to 97, and this region is identified in bPLs. The TAA stop codon was used in both bPRP-VIII and -IX, and appeared after the sequence TGC, which was present in other bPRPs except for bPRP-VI and bPLs that encode Cterminal cysteine residue [17]. The predicted 3D structures of bPRP-VIII and -IX mature region are shown in Fig. 5. The structural differences of $N$-glycosylation site, 
AATGCATGCTCTGTAATGGGTCTTCTCTTAAAAAATAAACACAGATGGTTTAGAAATGCAAAAAAAAAAAAAA

\section{Figure 2}

Nucleotide and deduced amino acid sequences of bPRP-IX. The arrow indicates the putative primary cleavage site of the signal peptide. The potential $\mathrm{N}$-glycosylation site is underlined with a dotted line. The asterisks indicate the termination codon. The polyadenylation signal is underlined with a solid line. 
bPRP-VIII bPRP-IX bPRP-I bPRP-II bPRP-III bPRP-IV bPRP-V bPRP-VI bPRP-VII bPL-Ala bPL-Val

bPRP-VIII bPRP-IX bPRP-I bPRP-II bPRP-III bPRP-IV bPRP-V bPRP-VI bPRP-VII bPL-Ala bPL-Val

bPRP-VIII bPRP-IX bPRP-I bPRP-II bPRP-III bPRP-IV bPRP-V bPRP-VI bPRP-VII bPL-Ala bPL-Val

bPRP-VIII bPRP-IX

bPRP-I

bPRP-II

bPRP-III

bPRP-IV

bPRP-V

bPRP-VI

bPRP-VII

bPL-Ala

bPL-Val
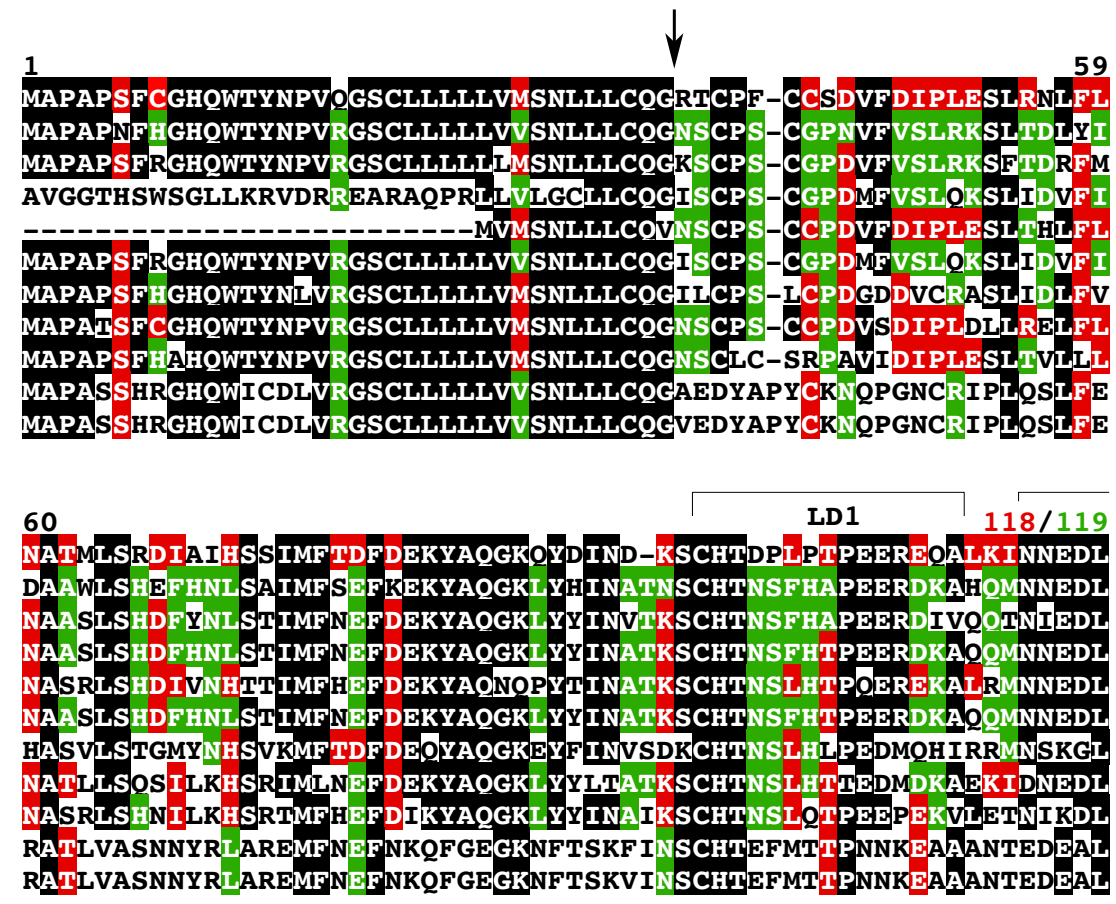

$119 / 120$ LD2 $178 / 179$

SKWI IMLLYSWKRPLNHLVTELONMKEVSDTILSSARONLKKVQELOEF IEROFSOI IFP SKWILVLLYSWNN PLYHLVPRLRNMKNLS EAVVSSAME I FNMSDKLQAF I ESOFRKVIVS SKWILVLLYSWNNPLHHLVTELOHMKELSNAFLSSATRF FNMSEKLQAF IEROFSK I IVP SKWILVLLYSWNNPLYYLLLELRNMKNLS FAVISSAME I FNMSEKLOAF I ESOFRK I I VP SKWILMLLYSWHRPLYLLVKDLOSMKEVSDTILSSAK ENMRK IE ELQAF IEROFSOVIYP SKWILVLLYSWNN PLYYLLLELRNMKNLS FAVISSAMII I FNSE KLQAF IESOFRK I IVP IMWI IMLLYSWQRPL YOLVTDLR SMKEVSNTILSSAR FNVKKLKELQALIERPFSOVIFT SKWILMLLYSWHRPLNHLVTDMRGMI EVSDTILSNAK ENVKKVE ELQAF IEROFCOI IIP SKWILMLLYSWNRPLYHLVTDLR SMKEVSDS IOSNARESVKNLLELOALIEROFCOI IFP LRLVISLLHSWDEPLHQAVTELLHRNGASPDILARAKEI IDKTKVLLEGVE--MIOKRVH LRLVISLLHSWDEPLHQAVTELLHRNGASPDILARA K I EDKTKVLLEGVE--MIOKRVH

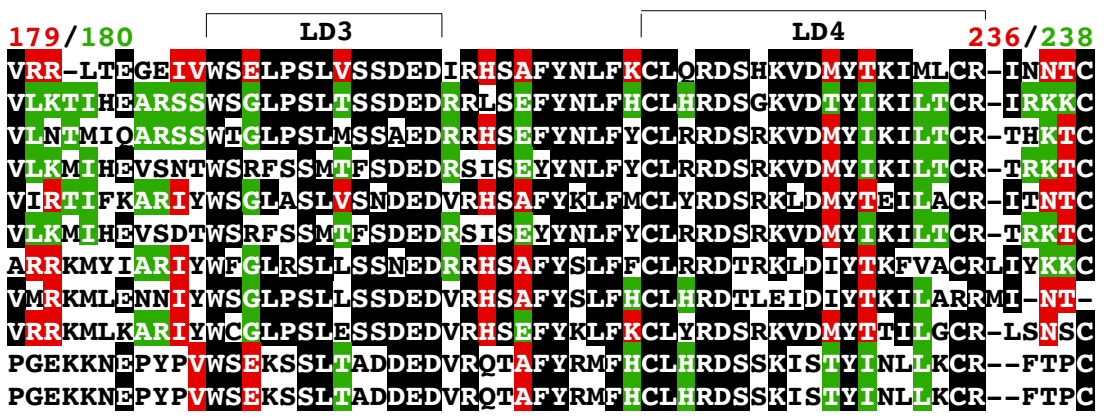

Figure 3

Comparison of amino acid sequences of bPRP-VIII and -IX with other members of bPRPs and bPLs. Residue present in both bPRP-VIII and -IX is shown in black boxes. Residue present in only bPRP-VIII is designated in red boxes. Residue present in only bPRP-IX is designated in green boxes. Amino acid sequences were aligned with assistance from Clustal W I.83 on the DDBJ web site. The arrow indicates the putative primary cleavage site of the signal peptide of bPRP-VII. LDI, 2, 3, and 4 refer to the conserved domain in the prolactin family. 


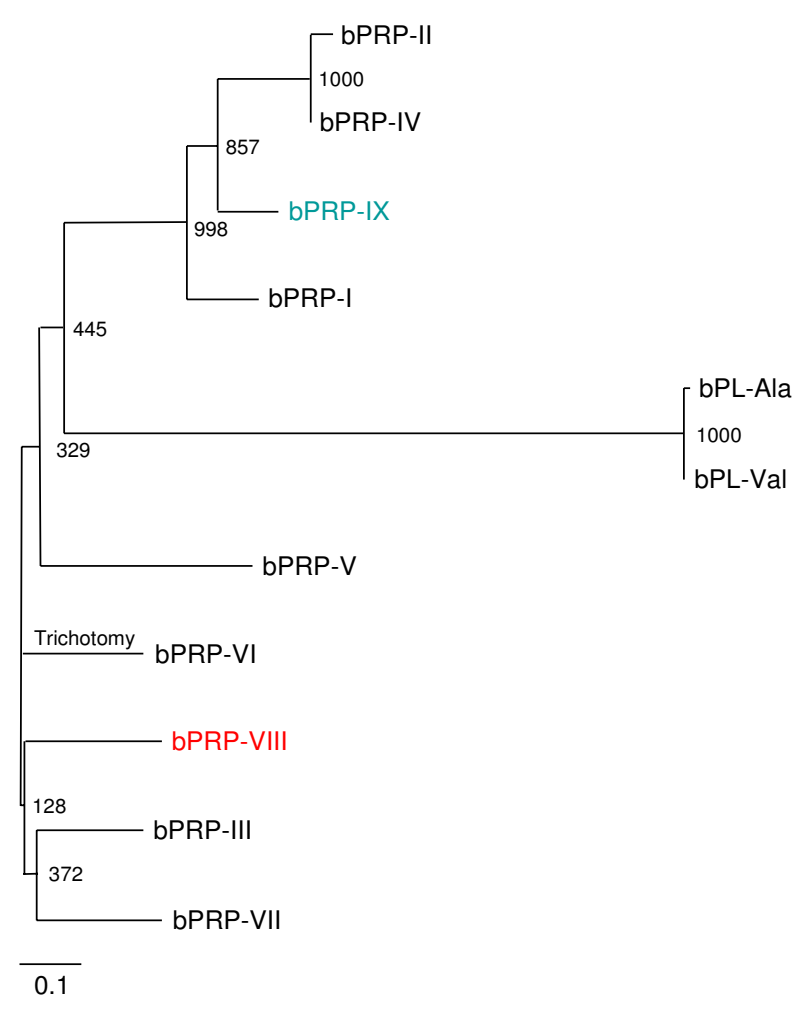

Figure 4

Phylogenetic tree of bPRPs and bPLs. The tree was constructed using TreeView following alignment of the protein sequences by the Clustal W I.83 algorithm. The numbers at the base of each branch division represent bootstrap values after 1000 repeats. Scale bar represents 0.1 amino acid replacements per amino acid site. For GenBank/DDBJ accession numbers, refer to Materials and Methods.

disulfide bond (-S-S-) and each atomic configuration were confirmed. We submitted these sequences to the DNA Data Bank of Japan (DDBJ). The DDBJ/GenBank accession Nos. are $\underline{\mathrm{AB} 196438}$ and $\underline{\mathrm{AB} 204881}$.

Expression of bPRP-VIII and -IX mRNA in bovine placenta The distribution of bPRP-VIII and -IX mRNA was examined in various bovine tissues by RT-PCR. Both bPRP mRNAs appeared only in the placental tissue (Fig. 6).

bPRP-VIII and -IX mRNA localization was determined by in situ hybridization in the bovine placentome on day 60 of gestation (Fig. 7). DIG-labeled bPRP-VIII and -IX antisense RNA probes specifically detected the mRNA transcript in the placentome and intercotyledonary membrane. Both $b P R P s$ appeared in the binucleate cells in the cotyledon and intercotyledonary membrane, and $b P R P-I X$ mRNA was also detected in trinucleate cells (Figs. 7D and $7 F)$. No significant signals were detected with sense probes in any genes (Fig. 7B and 7E).
Quantitative real-time RT-PCR analysis indicated that bPRP-VIII mRNA appeared primarily in the extra-embryonic membrane of Day 27, cotyledon and intercotyledonary membrane of Day 60 to 250 (Fig. 8). The expression intensity increased approximately four-fold in the cotyledon from Day 27 to Day 60, and then slightly decreased until Day 250 (approximately 0.6-fold). The expression intensity increased approximately three-fold in the intercotyledon from Day 27 to Day 60 and was maintained from Day 60 to Day 150. Then it then decreased slightly until Day 250 (approximately 0.7-fold). In the caruncular area, the expression increased by about four to five times by Day 60, and the expression level was maintained to late gestation. However no significant expression was detected in the intercaruncle. $b P R P-I X$ mRNA was also detected in the cotyledonary placentome and intercotyledonary membrane after Day 60 of gestation, but it did not appear in the extra-embryonic membrane on Day 27 of gestation. The mRNA expression intensity increased approximately 1.2-fold from Day 60 to Day 150 in the cotyledon and approximately 1.9-fold the intercotyledon, and then those values were maintained until Day 250. No expression of bPRP-IX was observed in the caruncular area on Day 27, but stable expression intensities were detected from Day 60 to Day 250. These expression intensities were rather strong compared to the intercotyledonary and cotyledonary areas, even though the sources for this gene may be binucleate and trinucleate cells.

The recombinant proteins of bPRP-VIII and -IX raised with HEK293 cell were detected by Western blot analysis (Fig. 9). The intense bands of bPRP-VIII with a FLAG epitope tag migrated to approximately $28 \mathrm{kDa}$ molecular weight, while those of bPRP-IX with a FLAG epitope tag migrated to $38 \mathrm{kDa}, 34 \mathrm{kDa}$ and $26 \mathrm{kDa}$ (Fig. 9).

\section{Discussion}

In the present study, we identified two new members of $b P R P$ cDNAs, bPRP-VIII and -IX. Totally nine bPRP genes have been identified at this moment in bovine.

bPRP-VIII and -IX cDNA sequences had following features, respectively. The position of the polyadenylation signal (AATAAA) in the bPRP-VIII started 20 bases upstream from the poly(A) additional site. This configuration is specific to bPRP-VIII. The number of bases in bPRP-VIII from the polyadenylation signal to poly(A) site are the fewest in known $b P R P s$. The position of the polyadenylation signal in the $b P R P-I X$ started 26 bases upstream from the poly(A) additional site. This position coincides with those in bPRP-II and bPRP-IV [14,15]. The signal peptide sequences in the $N$-terminal regions of both bPRP-VIII and -IX were conserved similarly to those in other bPRPs/ bPLs (Fig. 3). The signal cleavage site was predicted to be between the Glu-36 and Arg-37 in bPRP-VIII, and 

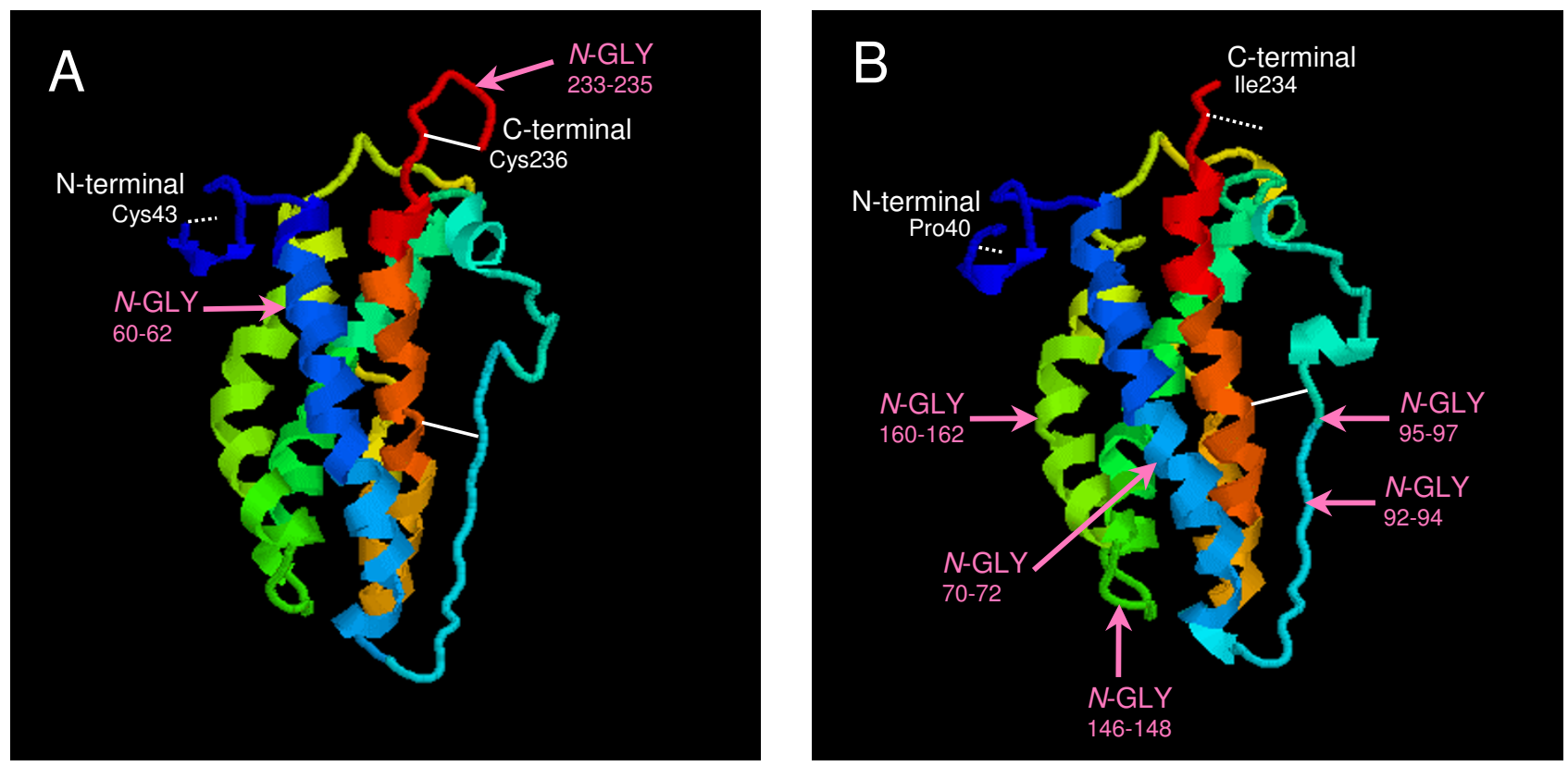

\section{Figure 5}

The predicted 3D structure of (A) bPRP-VIII and (B) bPRP-IX mature protein. The 3D structure were predicted by FAMS software. bPRP-VIII structure was able to construct from Cys43 to Cys236 amino acid region. bPRP-IX structure was able to construct from Pro40 to lle234 amino acid region. Disulfide bonds refer to white solid line. Predicted disulfide bonds refer to white dot line. N-GLY refer to potential N-glycosylation site.

between Glu-36 and Asn-37 in bPRP-IX by homology to the N-terminal regions of the bPRPs/bPLs (Fig. 3). As a result, bPRP-VIII is predicted to be a mature protein composed of 200 amino acids, while bPRP-IX has 202 amino acids. bPRP-VIII lacked two amino acids (positions 94 and 183), which is a sequence characteristic, since other known bPRPs have 202 amino acids. bPRP-VIII had two potential $N$-glycosylation sites (amino acid portions 60 to 62 and 233 to 235, Figs. 1, 3 and 5). The position of 60 to 62 coincided with those of bPRP-III, -VI, and -VII $[6,14,17]$, however, the second $N$-glycosylation site was found at 233 to 235 in amino acids. In contrast, bPRP-IX had four potential typical $N$-glycosylation sites of Asn-XSer/Thr (amino acid portions 70 to 72,92 to 94,146 to 148 , and 160 to 162 , Figs. 2, 3 and 5). All these configurations of Asn-X-Ser/Thr coincide with those of bPRP-II and -IV [14,15]. However, it is characteristic that only the bPRP-IX have atypical N-glycosylation site of Asn-X-Cys (amino acid portions 95 to 97). The conserved domains (LD1 to LD4, Fig. 3) were suggested by Yamakawa et al. [15] in other prolactin-related genes. The bPRP-VIII and IX sequences also revealed these LD domains. Cysteine residues (PRP-VIII positions 96, 213, and 230, and PRP-IX positions 97, 215, and 232) in the LD1 and LD4 domains were conserved in bPRPs/bPLs, except for bPRP-VI. bPRP-
VIII had four more cysteines (positions 38, 41, 42, and 236, Fig. 3, a total of seven residues in mature protein), and bPRP-IX had three more cysteines (positions 38, 41, and 238, Fig. 3, a total of six residues in mature protein).

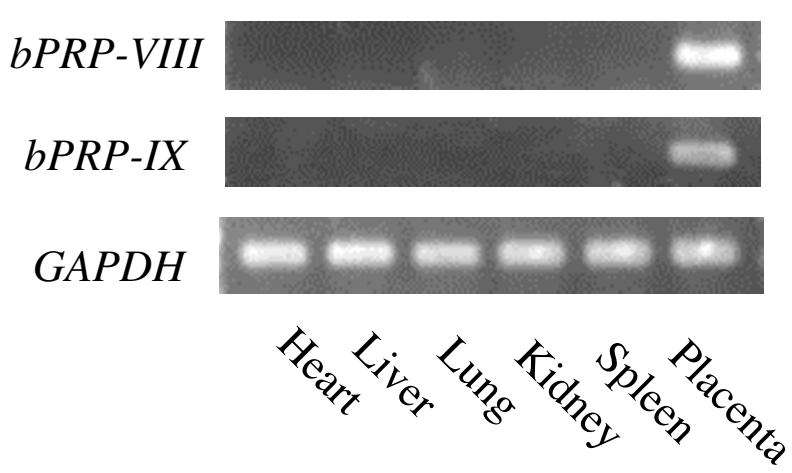

\section{Figure 6}

Expression of bPRP-VIII and -IX mRNA in bovine tissues. Heart, liver, lung, kidney, and spleen were used by RTPCR. Cotyledonary tissue at day I 48 of gestation was used as a placental sample. GAPDH expression in each tissue is presented as standard data. 

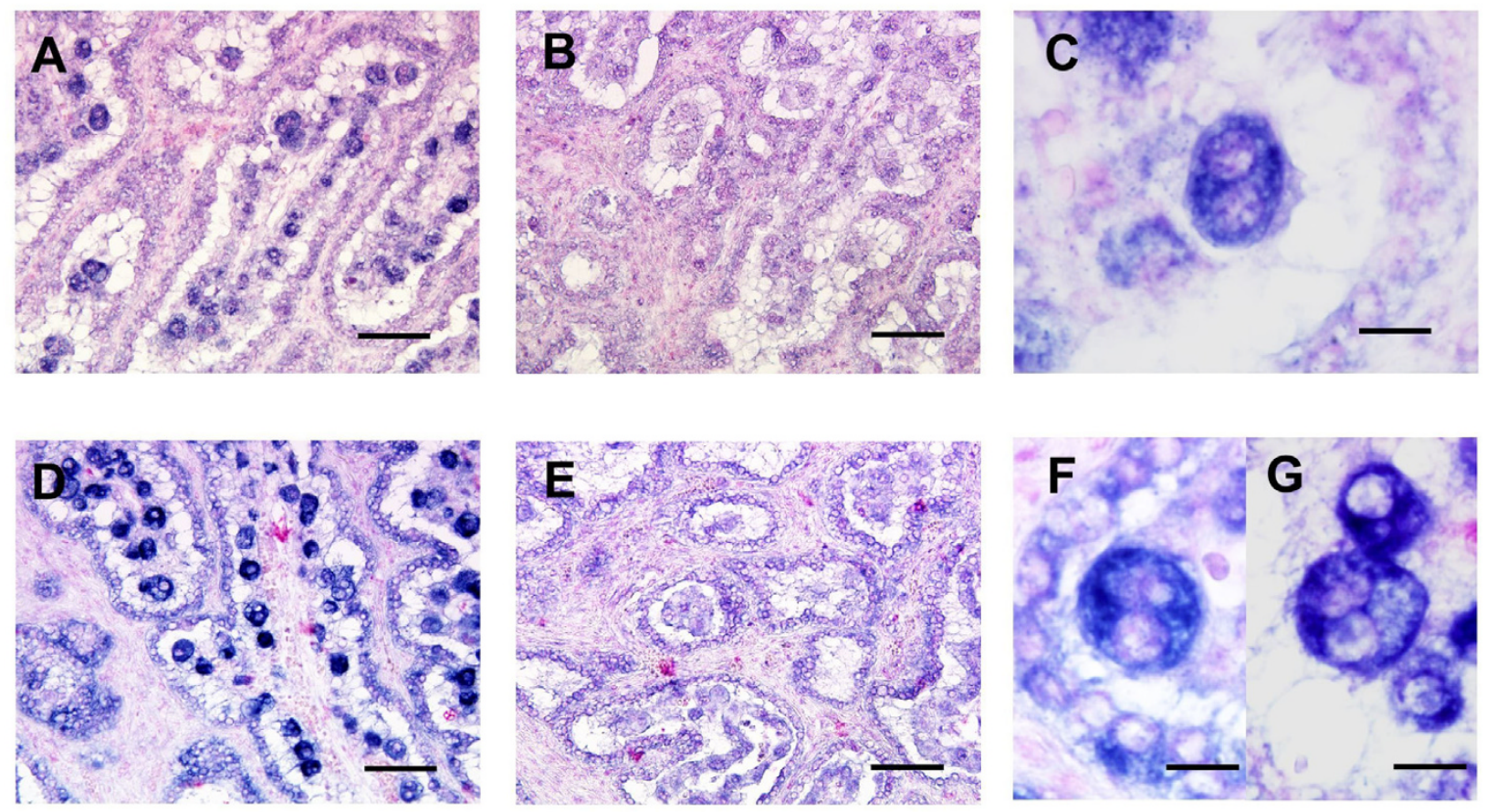

Figure 7

Localization of bPRP-VIII and -IX in the bovine placentome on day $\mathbf{6 0}$ of gestation. (A, B, C) bPRP-VIII and (D, E, F, G) bPRP-IX mRNA were detected by in situ hybridization. (A, C, D, F, G) DIG-labeled anti-sense cRNA probes were used. (B, E) DIG-labeled sense cRNA probes were used. Seven-microgram sections of bovine placentome were hybridized with each probes. Scale bar $=100 \mu \mathrm{m}$ on the $(A, B, D, E)$ and $20 \mu \mathrm{m}$ on the $(C, F, G)$.

These configurations indicate common features in bPRPs/ bPLs. In particular, the configuration of the cysteine residue in bPRP-VIII coincided exactly with that of bPRP-III in the mature sequence region. The cysteine residue in bPRPIX was also the same as that in bPRP-I, -II, and -IV in the mature sequence region. Therefore, both bPRP-VIII and IX may have three disulfide bonds similar to those of other bPRPs, except for bPRP-VII [6].

The primary mRNA expression of $b P R P$-VIII and -IX was observed in the binucleate cells (Fig. 7). Both genes could independently produce mature recombinant proteins in the mammalian cell expression system (Fig. 9). The binucleate cells may have produced the bPRP-VIII and -IX proteins simultaneously because bPRP-VIII and -IX mRNA expressed in the binucleate cells, and HEK293 cells translated these mRNA to each protein individually. Binucleate cells are also primary expression cells for $b P R P-I$ and $b P L$ $[27,28]$ and may have a specific function for implantation in the fetomaternal interface $[11,29]$. bPRP-VIII mRNA was also expressed in the extra-embryonic membrane just after the implantation period in the present study (Day 27 of gestation) (Fig. 8). bPRP-VIII may also be related to the implantation process like bPRP-I and -VII since bPRP-I was expressed during early gestation. Compare to these expression, no $b P R P-I X$ was present in the extra-embryonic membrane on Day 27 of gestation (Fig. 8). This mRNA expression pattern implies that bPRP-IX has a different role from $b P R P-I$ and $b P L$ in placental formation [6]. bPRP-IX mRNA was also detected in the trinucleate cells. A trinucleate cell is generated by migration of a binucleate cell to an endometrial epithelial cell with the progress of the pregnancy [30]. bPRP-IX may be a necessary molecule during middle to late gestation. Although both bPRP-VIII and -IX may be necessary molecules for gestation, in situ hybridization demonstrated that both genes were primarily expressed in binucleate and trinucleate cells. This means both genes derive from fetal side origin, but both were stably contained in the caruncular area throughout gestation, except bPRP-IX in early gestation. There are two potential causes of this result. One is contamination after separation. A second possibility is cell migration [30]. The present study and our previous study suggest that binucleate and trinucleate cells may migrate deeply into the endometrium after implantation and maintain their functions, which may be related to immu- 
Day 27

A

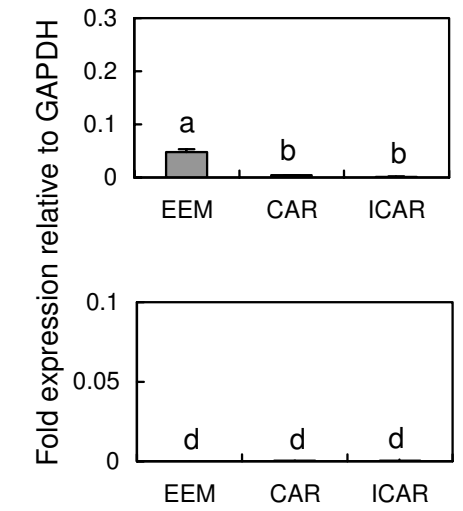

Day 60
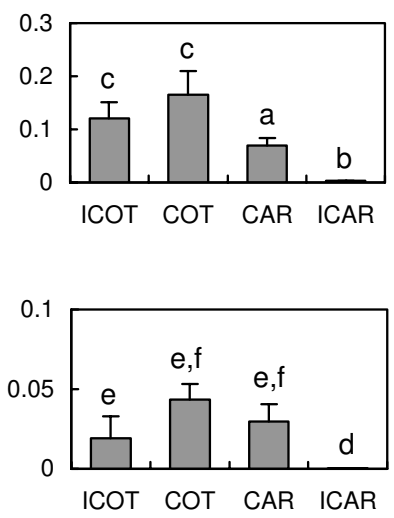

Day 150
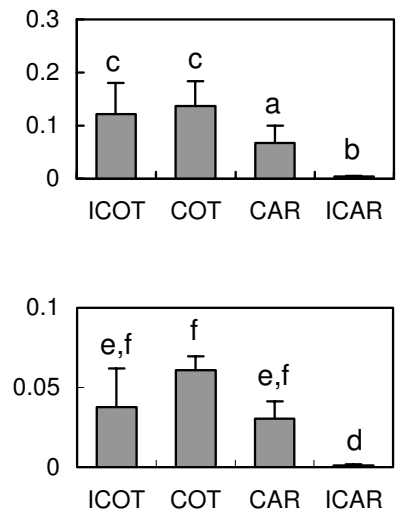

Day 250
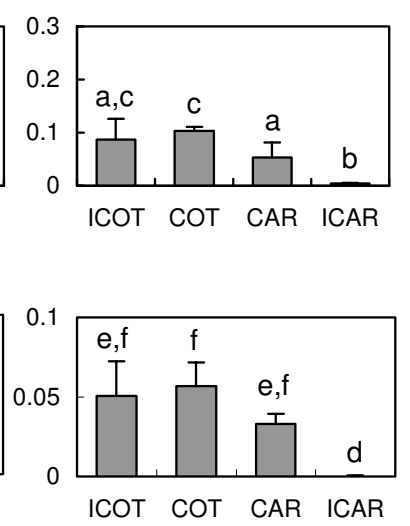

\section{Figure 8}

Quantitative real-time RT-PCR analysis of (A) bPRP-VIII and (B) bPRP-IX mRNA in bovine placenta. The total RNA was extracted from cotyledons containing extra-embryonic membrane (EEM), cotyledonary placenta (COT), intercotyledonary fetal membrane (ICOT), caruncular placenta (CAR), and intercaruncular endometrium (ICAR) on Day 27, Day 60, Day 150, and Day 250 of gestation. The expressions of these mRNAs were normalized to the expression of GAPDH measured in the same RNA preparation. Values are means \pm SD. Values with different letters are significantly different $(P<0.05)$.

nomodulation or stress during bovine gestation $[6,7,30,31]$. The molecular size of the recombinant proteins completely differed between bPRP-VIII and -IX. Transcripted bPRP-VIII protein is of relatively smaller size in the known bPRP family members, and the bPRP-IX protein is among the largest members $[6,32,33]$. The sugar chain addition in post-translational modification will give large effect in the difference of both molecular weights. It was found that the predicted 3D structure differed clearly in both molecules, because configuration of disulfide bonds and sugar chain addition dynamics are different. The tertiary structure of bPRP-VIII and -IX are similar to that of hPRL, so they may share the same receptor. However there is no information and the other evidence may not support this speculation. Only the bPRP-I gene has been known to produce protein in the placenta, however, the protein bound to the alpha2-macroglobulin [32], so it might have a biological function in paracrine status. Since all other bPRPs have a similar characteristic, it is difficult to say bPRPs are hormones secreted into circulation from the placenta, such as bPRL and bPL. It was possible to predict a part of their functions from the molecular weight, 3D structure, and post-translational modification. bPRP-VIII and -IX proteins may have different functions, since their temporal and special expressions were different. Various difficulties remain for understanding PRP functions, however, many of the same genes and molecules are expressed in bovine placenta as in rodent placenta [7].
Recently, bovine genome projects have addressed various shotgun sequence data and five known $b P R P s, b P L$, and $b P R L$, a total of seven prolactin related genes, hit in the NCBI genome database. They are located in Bos taurus chromosomes 12 and/or 23. bPRP-III, -VII and bPL were detected on chromosome 23 with $b P R L$. On the other hand, $b P R P-I,-V$, and $-V I$ are placed on chromosome 12. Rodents such as mice and rats also have various PLPs $[7,34]$, and they have been clustered on one chromosome, number 13 in mice and number 17 in rats $[34,35]$. bPRPIII and -VII were in comparatively close positions on the phylogenetic tree in Fig. 4, and they are clustered in the same chromosome 23. bPRP-VIII may be placed on chromosome 23 because bPRP-VIII was close to bPRP-III or VII on the phylogenetic tree, although the bootstrap value is low between bPRP-VIII and bPRP-III/-VII. bPRP-I, -V, and -VI, however, were comparatively close on the phylogenetic tree in Fig. 4; these genes were clustered on chromosome 12. bPRP-IX may be placed on chromosome 12 because bPRP-IX was close to bPRP-I on the phylogenetic tree. The actual configuration of the bPRP-VIII and -IX genome will be addressed in the future. Therefore, prolactin-related genes in mammals may have similar roles for placental function, but they may evolve through different phylogenetic processes, may be two separate pathways. For example, GH and PRL functions are different in various species, namely, GH uses the PRL receptor in some species, but not in others $[7,36]$.

In conclusion, we identified two new members of $b P R P s$, $b P R P$-VIII and -IX. bPRP-VIII was expressed in binucleate 


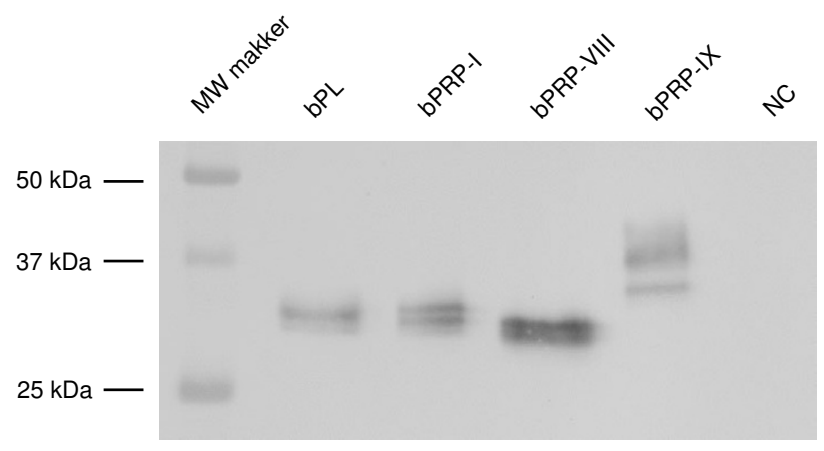

Figure 9

Production of recombinant bPRP-VIII, bPRP-IX, bPRP-I, and bPL proteins. Conditioned media from HEK 293 cells transiently transfected with each gene were collected, and the proteins $(10 \mu \mathrm{g})$ were loaded in separate lanes. The proteins were separated by SDS PAGE and specific proteins were detected by Western blot analysis using an anti-FLAG tag. MW Marker: molecular weight marker. NC: Negative control (transfected vector only).

cells in bovine trophoblast tissue and placentome. The expression appeared as well in $b P R P-I,-V I I$, and $b P L$, and it expressed from the implantation period to late in gestation. $b P R P-I X$ was expressed in binucleate cells and trinucleate cells, and was expressed somewhat late in gestation compared to other PRPs. These data indicate that various PRP genes in bovine placenta have coordination roles for gestation as evidenced in rodents.

\section{Acknowledgements}

The authors thank Prof. Yukio Tsunoda of Kinki University and Dr. Tomoyuki Tokunaga of the National Institute of Agrobiological Sciences for their help. This research was supported by a grant from the Bio-oriented Technology Research Advancement Institution (BRAIN), a grant of Research Project for Utilizing Advanced Technologies (05-1770), a grant-in-aid (HC04-226I-I) from the Ministry of Agriculture, Forestry, and Fisheries of Japan, grants (Hoga-kenkyu I6658105; Kiban-kenkyu C 17580284; Kibankenkyu B 17380172) from the Ministry of Education, Culture, Sport, Science, and Technology of Japan, and a grant of Animal Remodeling Project (05-20I) from National Institute of Agrobiological Sciences.

\section{References}

I. Dietz AB, Georges M, Threadgill DW, Womack JE, Schuler LA: Somatic cell mapping, polymorphism, and linkage analysis of bovine prolactin-related proteins and placental lactogen. Genomics 1992, 14:137-143.

2. Schuler LA, Kessler MA, Tanaka M, Nakashima K: Nomenclature clarification for the bovine placental prolactin-related hormones. Endocrinology 199I, I 26:2057.

3. Ushizawa K, Herath CB, Kaneyama K, Shiojima S, Hirasawa A, Takahashi T, Imai K, Ochiai K, Tokunaga T, Tsunoda Y, Tsujimoto G, Hashizume K: cDNA microarray analysis of bovine embryo gene expression profiles during the pre-implantation period. Reprod Biol Endocrinol 2004, 2:77.

4. Kessler MA, Duello TM, Schuler LA: Expression of prolactinrelated hormones in the early bovine conceptus, and potential for paracrine effect on the endometrium. Endocrinology |99|, | 29:|885-|895.
5. Yamada O, Todoroki J, Kizaki K, Takahashi T, Imai K, Patel OV, Schuler LA, Hashizume K: Expression of prolactin-related protein $I$ at the fetomaternal interface during the implantation period in cows. Reproduction 2002, I 24:427-437.

6. Ushizawa K, Kaneyama K, Takahashi T, Tokunaga T, Tsunoda Y, Hashizume $\mathrm{K}$ : Cloning and expression of a new member of prolactin-related protein in bovine placenta: bovine prolactin-related protein-VII. Biochem Biophys Res Commun 2005, 326:435-44I.

7. Soares MJ: The prolactin and growth hormone families: Pregnancy-specific hormones/cytokines at the maternal-fetal interface. Reprod Biol Endocrinol 2004, 2:5I.

8. Muller H, Liu B, Croy BA, Head JR, Hunt JS, Dai G, Soares MJ: Uterine natural killer cells are targets for a trophoblast cell-specific cytokine, prolactin-like protein-A. Endocrinology 1999, | 40:27| |-2720.

9. Ain R, Tash JS, Soares MJ: Prolactin-like protein-A is a functional modulator of natural killer cells at the maternal-fetal interface. Mol Cell Endocrinol 2003, 204:65-74.

10. Lin J, Linzer DIH: Induction of megakaryocyte differentiation by a novel pregnancy-specific hormone. J Biol Chem 1999, 274:2|485-2|489.

II. Bittorf T, Jaster R, Soares MJ, Seiler J, Brock J, Friese K, Muller H: Induction of erythroid proliferation and differentiation by a trophoblast-specific cytokine involves activation of the JAK/ STAT pathway. J Mol Endocrinol 2000, 25:253-262.

12. Bhattacharyya S, Lin J, Linzer DIH: Reactivation of a hematopoietic endocrine program of pregnancy contributes to recovery from thrombocytopenia. Mol Endocrinol 2002, I 6: | 386-1393.

13. Schuler LA, Hurley WL: Molecular cloning of a prolactin-related mRNA expressed in bovine placenta. Proc Natl Acad Sci USA 1987, 84:5650-5654.

14. Kessler MA, Milosavljevic M, Zieler CG, Schuler LA: A subfamily of bovine prolactin-related transcripts distinct from placental lactogen in the fetal placenta. Biochemistry I989, 28:5I54-5 I6I.

15. Yamakawa M, Tanaka M, Koyama M, Kagesato $Y$, Watahiki M, Yamamoto $M$, Nakashima K: Expression of new members of the prolactin growth hormone gene family in bovine placenta. Isolation and characterization of two prolactin-like cDNA clones. J Biol Chem 1990, 265:89| 5-8920.

16. Tanaka M, Yamakawa M, Watahiki M, Yamamoto M, Nakashima K: Isolation of a novel prolactin-like cDNA clone from bovine placenta: occurrence of new family members. Biochim Biophys Acta 1989, 1008:193-197.

17. Tanaka M, Minoura $H$, Ushiro $H$, Nakashima K: A novel cDNA clone encoding a prolactin-like protein that lacks the two Cterminal cysteine residues isolated from bovine placenta. Biochim Biophys Acta 1991, 1088:385-389.

18. Saitou N, Nei N: A neighbor-joining method: a new method for constructing phylogenetic tree. Mol Biol Evol 1987, 44:406-425.

19. Page RDM: TREEVIEW: An application to display phylogenetic trees on personal computers. Comput Appl Biosci 1996, I 2:357-358.

20. Ogata K, Umeyama $\mathrm{H}$ : An automatic homology modeling method consisting of database searches and simulated annealing. I Mol Graphics Mod 2000, I 8:258-272.

2I. Bernstein HJ: Recent changes to RasMol, recombining the variants. Trends Biochem Sci 2000, 25:453-455.

22. Herath $C B$, Shiojima S, Ishiwata $H$, Katsuma S, Kadowaki T, Ushizawa K, Imai K, Takahashi T, Hirasawa A, Tsujimoto G, Hashizume K: Pregnancy-associated changes in genome-wide gene expression profiles in the liver of cow throughout pregnancy. Biochem Biophys Res Commun 2004, 3 I 3:666-680.

23. Kizaki K, Yamada O, Nakano H, Takahashi T, Yamauchi N, Imai K, Hashizume K: Cloning and localization of heparanase in bovine placenta. Placenta 2003, 24:424-430.

24. Kizaki K, Nakano H, Nakano H, Takahashi T, Imai K, Hashizume K: Expression of heparanase mRNA in bovine placenta during gestation. Reproduction 200 I, I 2 I:573-580.

25. Laemmli UK: Cleavage of structural proteins during the assembly of the head of bacteriophage T4. Nature 1970, 227:680-685.

26. Towbin H, Staehelin T, Gordon J: Electrophoretic transfer of proteins from polyacrylamide gels to nitrocellulose sheets: procedure and some applications. Proc Natl Acad Sci USA 1979, 76:4350-4354. 
27. Milosavljevic M, Duello TM, Schuler LA: In situ localization of two prolactin-related messenger ribonucleic acids to binucleate cells of bovine placentomes. Endocrinology 1989, I 25:883-889.

28. Nakano H, Takahashi T, Imai K, Hashizume K: Expression of placental lactogen and cytokeratin in bovine placental binucleate cells in culture. Cell Tissue Res 200I, 303:263-270.

29. Anthony RV, Liang R, Kayl EP, Pratt SL: The growth hormone/prolactin gene family in ruminant placentae. J Reprod Fertil Suppl 1995, 49:83-95.

30. Wooding FBP, Beckers JF: Trinucleate cells and the ultrastructural localisation of bovine placental lactogen. Cell Tissue Res 1987, 247:667-673.

31. Ain R, Dai G, Dunmore JH, Godwin AR, Soares MJ: A prolactin family paralog regulates reproductive adaptations to a physiological stressor. Proc Nat Acad Sci USA 2004, I 0 I: I6543-I 6548.

32. Kessler MA, Schuler LA: Purification and properties of placental prolactin-related protein-I. Placenta 1997, I 8:29-36.

33. Takahashi T, Aso H, Hashizume K: Immunological and biological activities of bovine placental lactogen in placental explant culture. J Reprod Dev 200I, 47:63-67.

34. Wiemers DO, Shao LJ, Ain R, Dai G, Soares MJ: The mouse prolactin gene family locus. Endocrinology 2003, I 44:3 I 3-25.

35. Öztürk A, Fresnoza A, Savoie A, Duckworth HW, Duckworth ML: Defining regulatory regions in the rat prolactin gene family locus usinga large PI genomic clone. Endocrinology 2003, I 44:4742-4754.

36. Talamantes F, Ogren L, Markoff E, Woodard S, Madrid J: Phylogenetic distribution, regulation of secretion, and prolactin-like effects of placental lactogens. Fed Proc 1980, 39:2582-2587.

\section{Publish with Biomed Central and every scientist can read your work free of charge}

"BioMed Central will be the most significant development for disseminating the results of biomedical research in our lifetime. "

Sir Paul Nurse, Cancer Research UK

Your research papers will be:

- available free of charge to the entire biomedical community

- peer reviewed and published immediately upon acceptance

- cited in PubMed and archived on PubMed Central

- yours - you keep the copyright

Submit your manuscript here:

http://www.biomedcentral.com/info/publishing_adv.asp
BiolMedcentral 